\title{
Cytolytic vaginosis: misdiagnosed as candidal vaginitis
}

\author{
Nilgun Cerikcioglu ${ }^{1}$ and M. Sinan Beksac ${ }^{2}$ \\ ${ }^{1}$ Department of Microbiology, Marmara University School of Medicine, Turkey \\ ${ }^{2}$ Gynecology and Obstetrics Department, Hacettepe University Hospital, Turkey
}

Objectives: In this study, 210 women with vaginal discharge and other symptoms/signs of genital pathology suggestive of vulvovaginal candidiasis (VVC) were involved in order to distinguish true WVC and cytolytic vaginosis $(\mathrm{CV})$ cases.

Methods: Fungal cultures, $10 \%$ potassium hydroxide $(\mathrm{KOH})$ and $\mathrm{Gram}$ stained preparations and $\mathrm{pH}$ measurements were performed on the vaginal discharge material of each patient.

Results: Fifteen patients (7.1\%) were diagnosed with cytolytic vaginosis according to their clinical and microbiological findings, including abundant lactobacilli, fragmented epithelial cells and/or free nuclei due to cytolysis, seen in their discharge materials on microscopic examination, but no fungal growth.

Conclusions: The results of this study may contribute to the reports in the literature indicating the importance of such disorders, which are generally misdiagnosed as candidiasis.

Key words: VAginal Discharge; Dyspareunia; Dysuria; ItCHING

\section{INTRODUCTION}

In healthy women Lactobacilli are predominant organisms in the cervix and vagina. They maintain the acidic $\mathrm{pH}(4.0-4.5 \%)$ of the vagina by producing lactic acid, thereby protecting the vagina against infections ${ }^{1}$.

Lactobacilli may control the overgrowth of E.coli, Candida spp., Gardnerella vaginalis and Mobilincus spp. by producing $\mathrm{H}_{2} \mathrm{O}_{2}$ and other inhibitory substances such as acidolin, and by competing for nutrients ${ }^{2-4}$.

In some women, who are still in the reproductive stage of life, an abundant growth of lactobacilli may result in lysis of vaginal epithelial cells ${ }^{5,6}$. This change in the vaginal microenvironment may lead to symptoms such as pruritus, burning and abundant severe whitish discharge, similar to symptoms of candidal vaginitis $^{5}$. On microscopic examination, large amounts of lactobacilli and fragmented or lysed epithelial cells are observed; thus the disorder is called 'cytolytic vaginosis' (CV) in the literature ${ }^{7}$.

This study was carried out to assess CV cases in a group of patients with symptoms and signs suggestive of vulvovaginal candidiasis (VVC).

\section{MATERIALS AND METHODS}

\section{Patients}

In this study, 210 women that were all within the reproductive age group, exhibiting symptoms of abnormal vaginal discharge, pruritus, burning and dyspareunia or signs of inflammation of the genital tract suggestive of VVC, were clinically and microbiologically evaluated. Vaginal dis-

Correspondence to: Nilgun Cerikcioglu, PhD, Assist. Prof. Dr., Marmara University, School of Medicine, Department of Microbiology, Tibbiye Street, 34668, Haydarpasa Campus, Istanbul, Turkey. Email: nilguncerik@yahoo.com 
charge materials from the vaginal side wall and/or the pool were obtained by using cotton swabs after the insertion of a speculum.

\section{Microbiological studies}

Three samples were obtained from each patient's discharge material by using sterile swabs. The three swabs were placed in Stuart's transport medium.

One swab was used to assess $\mathrm{pH}$ of the discharge by means of $\mathrm{pH}$ indicator paper (Merck, Germany), immediately after collecting the sample. The second swab was used for inoculation on Sabouraud dextrose agar (SDA) plates, which were incubated at $35^{\circ} \mathrm{C}$ for 3 days. The third swab was mixed with $10 \% \mathrm{KOH}$ as a wet mount and Gram stained preparation, in order to look for the presence of yeast cells and/ or pseudohyphae or disrupted epithelia and abundant lactobacilli as indicators of VVC and $\mathrm{CV}$, respectively ${ }^{7,8}$.

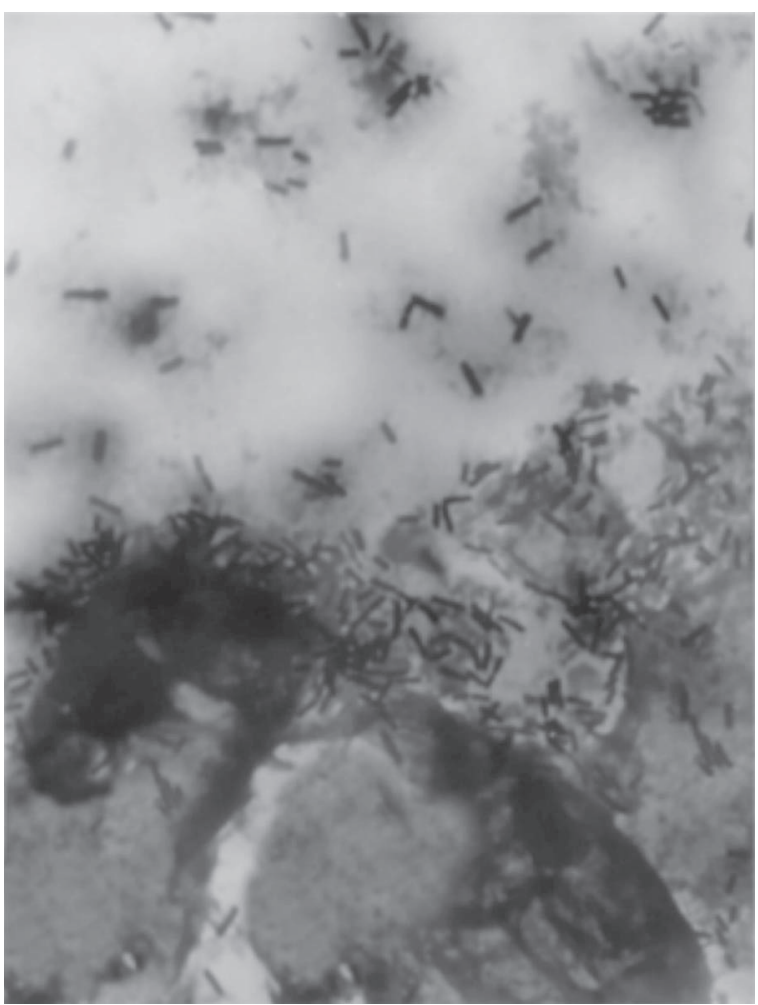

Figure I Lactobacilli and disrupted epithelial cells

\section{RESULTS}

During the study period 210 women suggestive of VVC were evaluated. None of these women were pregnant, and only one had diabetes mellitus which was under control. All of these patients were suffering from a whitish vaginal discharge. The other complaints and signs included malodor in 33 patients $(15.7 \%)$, pruritus in 32 patients $(15.2 \%)$, erythema and edema of vulva or vagina in 21 patients (10\%), burning in 15 patients $(7.1 \%)$ and dyspareunia in nine patients $(4.3 \%)$.

Twenty-seven (12.8\%) individuals were diagnosed as having active VVC confirmed by cultures on SDA, and/or microscopy, while 15 patients $(7.1 \%)$ did not have any positive culture results for yeasts on SDA, but on Gram stained preparations of their vaginal discharges, abundant lactobacilli and many fragmented epithelial cells and/or free nuclei were observed (Figures 1 and 2). Depending on the clinical and microbiological findings stated in the literature, all were diagnosed

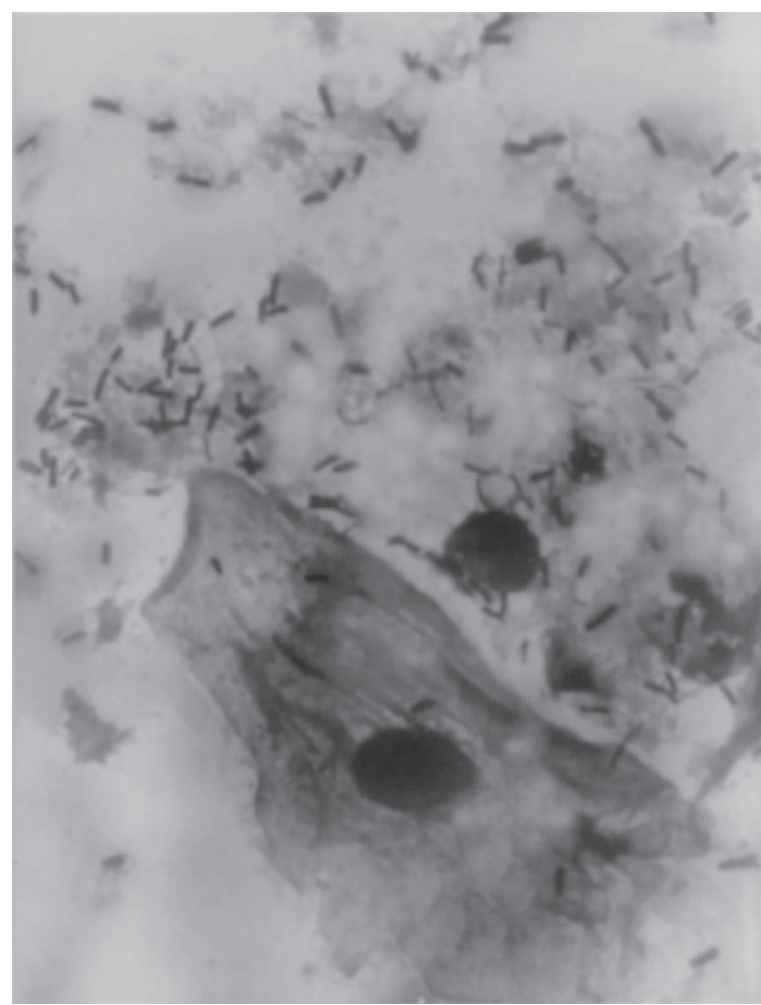

Figure 2 Lactobacilli and free nucleus of a lysed epithelium 
as having $\mathrm{CV}^{5}$. Leucocytes were not observed in samples belonging to the patients diagnosed as having CV; except for two samples which revealed a few leucocytes in the microscopic field. A $\mathrm{pH}$ of $4.0-4.5$ was detected in all discharge samples.

All of these 15 cases were in the reproductive age group of $25-40$ years. Five $(33.3 \%)$ were in the luteal phase, with enhanced complaints of discharge and pruritus.

\section{DISCUSSION}

Normal vaginal flora in adult women within the reproductive age group is usually dominated with lactobacilli. Lactobacilli in low numbers (five bacilli per 10 squamous cells) in vaginal discharges have been defined as protective factors against vaginal candidiasis ${ }^{9}$.

According to several studies lactobacilli build up a barrier against candidal overgrowth by blocking the adhesion of yeasts to epithelial cells through competition for nutrients ${ }^{10-13}$. However, among individuals within the reproductive age group some may develop $\mathrm{CV}$ due to an excess amount of lactobacilli. In such cases, lactobacilli alone or with other bacteria may cause dissolution of the vaginal intermediate epithelium that may result in the presence of free nuclei. This dissolution may be responsible for complaints of dysuria $^{5}$.

Fifteen patients had severe vaginal discharge and/or additional manifestations suggestive of VVC. Most of them had a history of failure of repeated antifungal therapy regimens for suspected recurrent VVC (RVVC), indicating that the clinical picture was non-candidal vaginitis and was misdiagnosed. Indeed, microscopy revealed disrupted squamous epithelial cells and/or free nuclei due to cytolysis and an abundant number of lactobacilli, instead of any fungal structures. Another remarkable point was the absence of leucocytes on Gram preparations in all but two of the samples.

One of our patients had diabetes mellitus. Some authors claim that the lactobacilli are more abundant in women with high serum glucose levels ${ }^{10}$. We also detected that five $(33.3 \%)$ of our patients who had complaints of increased dis- charge and itching were in their luteal phase. It has been suggested that in the luteal phase there is a remarkable rise in the number of colonizing lactobacilli ${ }^{4}$. After a correct diagnosis, the treatment may be directed toward reducing the number of lactobacilli by elevating the $\mathrm{pH}$ with alkaline douches such as Na-bicarbonate solution $^{5,7}$. The two patients who received the treatment benefited from it.

The clinical condition called CV was previously known as 'lactobacillus overgrowth syndrome' or 'Doderlein's cytolysis', because the typical disruption of epithelial cells is associated with the digestive activity of lactobacilli ${ }^{4,14}$. Although an old phenomenon, the exact mechanisms leading to fragmentation or cytolysis of vaginal epithelia in those women are not known. Abundant lactobacilli covering the fragmented epithelial cells may be confused with the "clue cells' of bacterial vaginosis (BV), these are therefore called 'false clue cells". In the literature, we found no studies that mentioned a confusion between $\mathrm{BV}$ and $\mathrm{CV}$ according to the microscopic appearance. BV may be diagnosed by $\mathrm{pH}$ measurements and 'whiff' tests. All of our cases yielded acidic $\mathrm{pH}(4.0-4.5)$ with negative whiff tests, which were sufficient for ruling out BV. In one study, the number of patients diagnosed with $\mathrm{CV}$ was defined as only five in 101 women with abundant vaginal discharge ${ }^{15}$. In our study there were 210 patients with remarkable vaginal discharge and the detected $\mathrm{CV}$ cases were 15 (7.1\%), which is in agreement with the previous finding.

The diagnosis of $\mathrm{CV}$ seems to bother clinicians and patients because it also mimics VVC and most of the cases that are accepted as 'chronic VVC' which is unresponsive to antifungal drugs, are waiting for the correct medical approach $^{16}$. However in one study, of 271 patients with vulvovaginal complaints, 29 $(10.7 \%)$ were diagnosed as having suggestive VVC, but only $16(5.9 \%)$ had a confirmed diagnosis of candidiasis ${ }^{14}$. Although not reported in the article, the remaining 13 cases could have been diagnosed as $\mathrm{CV}$, if the authors had searched for that condition.

While VVC is accepted as an important genital disorder comprising $10-30 \%$ of all vulvovaginal 
pathologies with discharge, $\mathrm{CV}$, with a proportion of $5-7 \%$ in the same patient population, must not be disregarded, but is to be taken as a significant clinical condition.

According to data given in the literature, most patients have been treated with antifungal medications, although Candida spp. has not been identified $^{7,17}$.

In conclusion, the differentiation of CV from VVC and other similar disorders would prevent the clinician from giving inappropriate and useless treatment to the patient.

\section{CONCLUSIONS}

1. In this study the rate of $\mathrm{CV}$ cases among women with suggestive VVC was found to be $7.1 \%$.

2. The results of this study, much like the previous studies in the literature, illustrate the importance of being careful when evaluating women with presumed vaginal candidiasis. A misdiagnosis can lead to the patient pestering their gynecologist again and again in order to resolve their complaint, and means that they are originally treated with drugs that are useless for CV.

\section{REFERENCES}

1. Galask RP, Larsen B, Ohm MJ. Vaginal flora and its role in disease entities. Clin Obstet Gynecol 1976;19:61-81

2. Hughes V1, Hiller SL. Microbiologic characteristics of lactobacillus products used for colonization of the vagina. Obstet Gynecol 1990;75:245-8

3. Mardh PA. The vaginal ecosystem. Am J Obstet Gynecol 1991;165:1163-8

4. Sharp HC, Kronh MA, Klebanoff SJ, et al. The relationship of hydrogen peroxide producing lactobacilli vaginosis and genital microflora in pregnant women. Obstet Gynecol 1992;79:369-73

5. Cibley LJ, Cibley LJ. Cytolytic vaginosis. Am J Obstet Gynecol 1991;165:1245-8

6. Nasiell K, Nasiell M, Hjerpe A, et al. The occurence of bacillus vaginalis Doderlein and cytolysis in dysplasia, carcinoma, and invasive carcinoma of the uterine cervix. Acta Cytol 1972;16:215

7. Paavonen J. Vulvodynia-a complex syndrome of vulvar pain. Acta Obstet Gynecol Scand 1995; 74:243-7

8. Sobel JD. Pathogenesis and epidemiology of vulvovaginal candidiasis. Ann New York Acad Sci 1988;544:547- 57
9. Osset J, Garcia E, Bartolome RM, et al. Role of lactobacillus as protector against vaginal candidiasis. Med Clin (Barc) 2001;22:285-8

10. Carvalho G. Döderlein bacilli in vaginal smears of post-menopausal women. Acta Cytopathol 1966;10:286-8

11. Sobel JD. Epidemiology and pathogenesis of recurrent vulvovaginal candidiasis. Am J Obstet Gynecol 1985; 152:924-35

12. Sobel JD. Pathogenesis and treatment of recurrent vulvovaginal candidiasis. Clin Infect Dis 1992;14(Supp 1):48 - 53

13. Sobel JD. Candidal vulvovaginitis. Clin Obstet Gynecol 1993;16:153-87

14. Cibley LJ, Cibley LJ, Baldwin D. Diagnosing candidiasis. A new, cost-effective technique. J Reprod Med 1998;43:925-8

15. Wathna B, Holst E, Hovelius B, et al. Vaginal discharge. Comparison of clinical, laboratory and microbiological finings. Acta Obstet Gynecol Scand 1994;73:802-8

16. Kinghorn GR. Vulvovaginal candidosis. J Antimicrob Chemother 1991;28(Suppl A):59-66

17. Witkin SS. Immunology of the vagina. Clin Obstet Gynecol 1993;36:122-144

ReCeIVed 08/15/03; ACCEPTED 11/14/03 




The Scientific World Journal
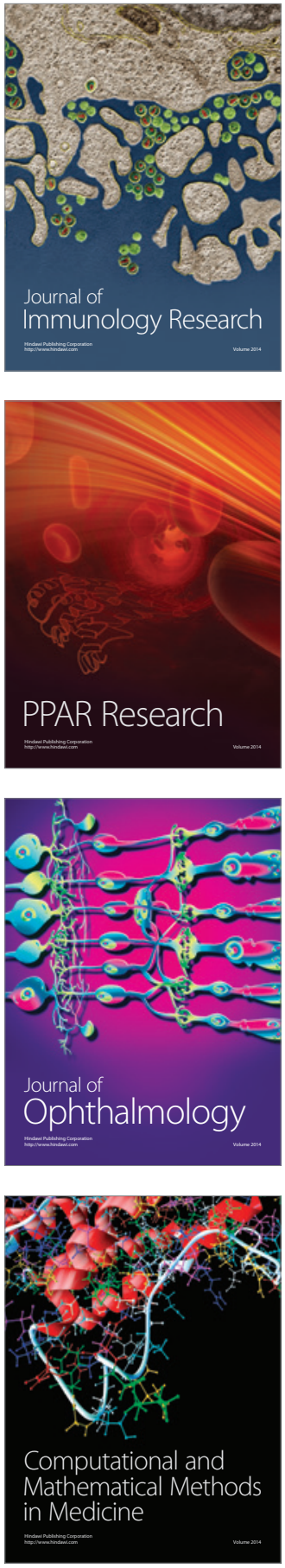

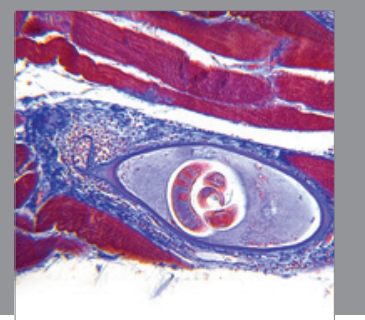

Gastroenterology

Research and Practice
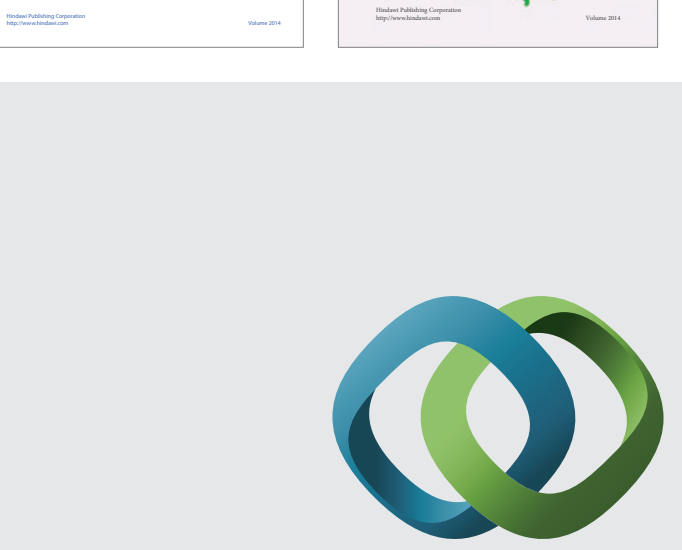

\section{Hindawi}

Submit your manuscripts at

http://www.hindawi.com
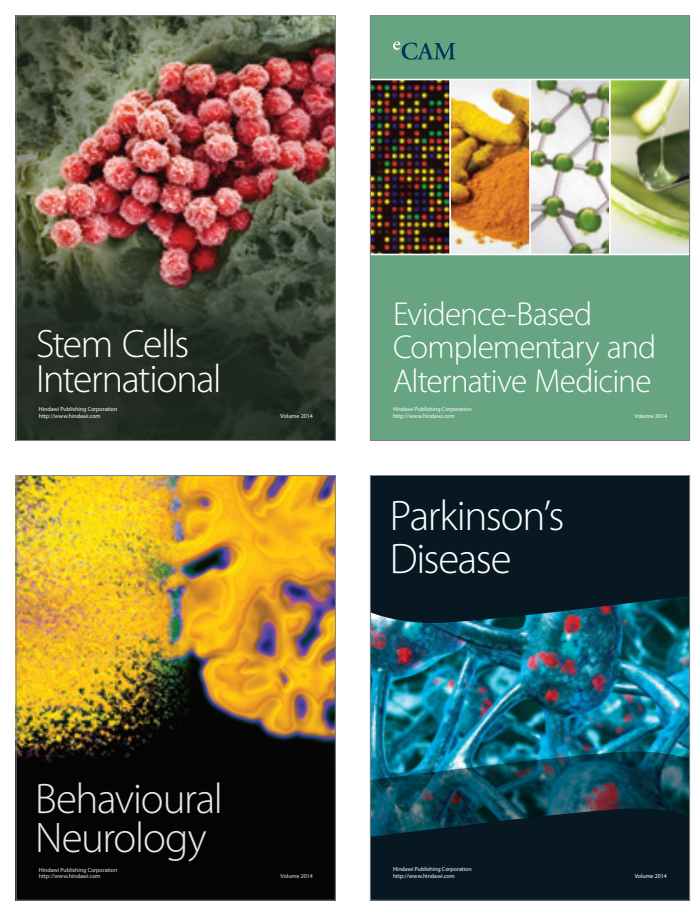

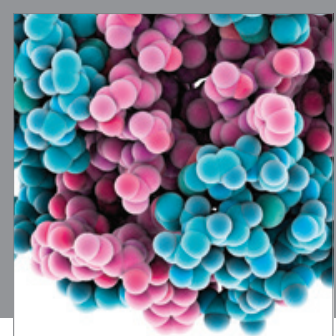

Journal of
Diabetes Research

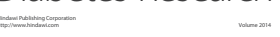

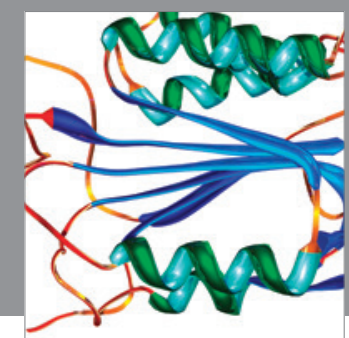

Disease Markers
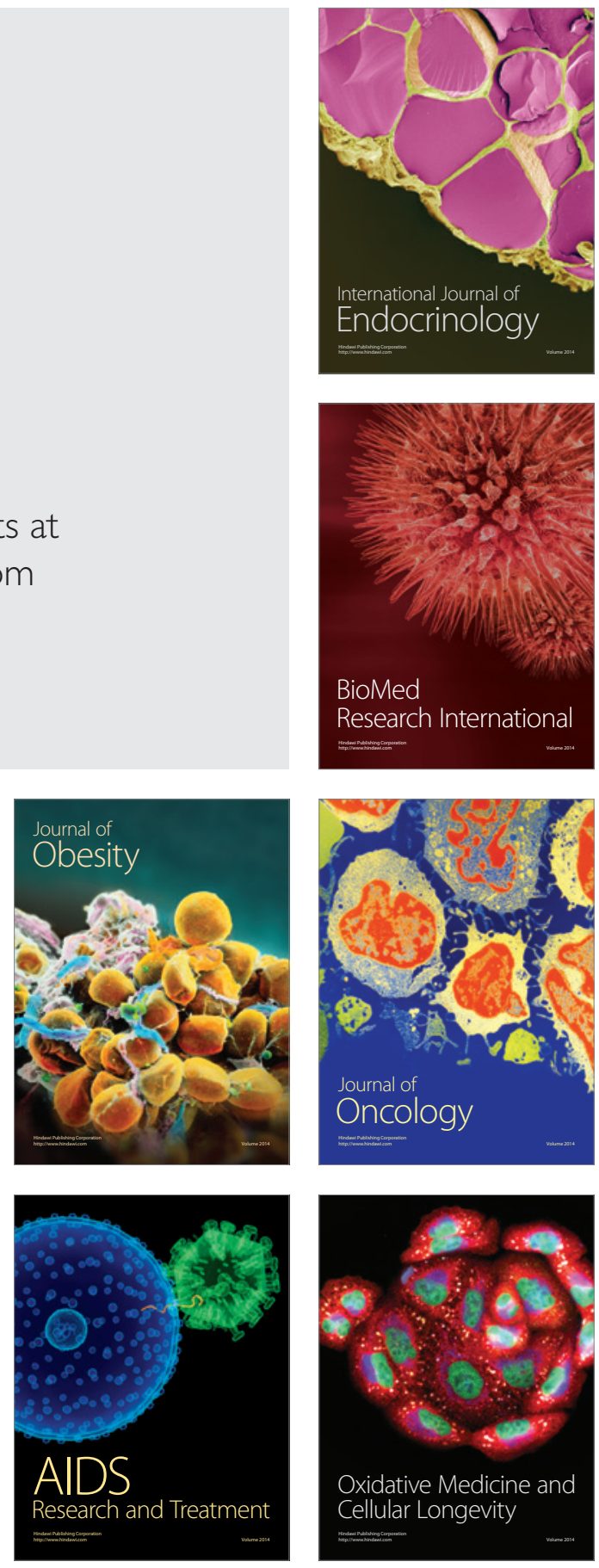